\title{
On Frequency Domain Adaptive Filters using the Overlap-add Method
}

\author{
P.C.W. Sommen ${ }^{*}$ and J.A.K.S. Jayasinghe \# \\ * Philips Research Laboratories, P.O.Box 80000, 5600 JA Eindhoven, The Netherlands \\ \# Technical University Twente, P.O.Box 217, 7500 AE Enschede, The Netherlands
}

\begin{abstract}
Frequency Domain Adaptive Filters (FDAFs) are an important subject of current research in digital signal processing. One of the main reasons is that FDAFs have the ability to reduce complexity, especially when the filter length becomes very large. In Adaptive Filter $(A F)$ configurations a convolution and a correlation have to be carried out. These operations can be done by using overlap methods in combination with Discrete Fourier Transforms (DFTs). Two well-known overlap methods are the so-called overlap-save and the overlap-add method. In literature $[1]$ it is stated that for $A F$ configurations the overlap-save method is to be preferred to the overlap-add method for complex. ity reasons. By introducing a new FDAF structure we will show that this statement is not correct and that an FDAF using the overlap-add method can be realized with the same number of DFTs as the FDAF using the overlap-save method.
\end{abstract}

\section{Introduction}

Adaptive Filters (AF) are extremely useful devices in many applications of digital signal processing. In this paper we will restrict ourselves for simplicity to the acoustic echo cancellation problem of which the basic scheme is given in Fig.1. Signal $x(k)$ is reflected via an acoustic echo path as an echo signal $e(k)$. Together with the speech signal $s(k)$, this signal $e(k)$ arrives at a microphone. The AF has to perform a linear convolution between the input signal $x(k)$ and the adaptive weights to make a replica $\hat{e}(k)$ of the echo signal $e(k)$. Furthermore the adaptive weights are updated as long as there is correlation between the input signal $x(k)$ and the residual signal $r(k)=s(k)+e(k)-\hat{e}(k)$. In steady state this residual signal will almost be equal to the signal $s(k)$.

In this paper we will restrict ourselves to the Frequency Domain Adaptive Filter (FDAF), where the transformation between time and frequency domain is carried out by a Discrete Fourier Transform (DFT). In this FDAF configuration the linear convolution (correlation) is accomplished by a circular convolution (correlation) using special overlap methods. Two well-known overlap methods are the overlap-save and the overlap-add method. In [1] it is stated that for FDAF configurations the overlap-save method is preferable

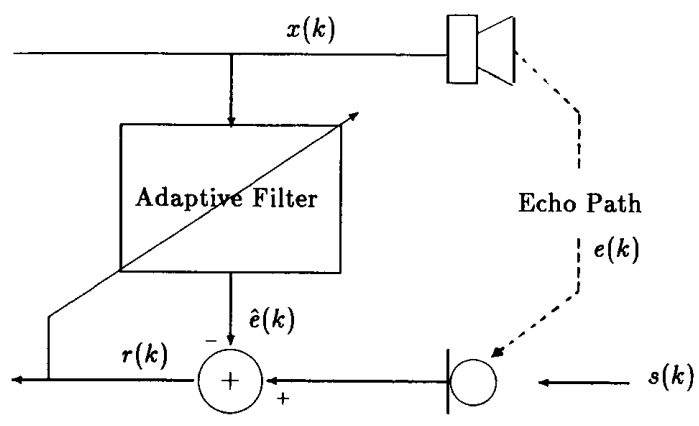

Fig. 1 Adaptive Filter in an acoustic environment.

to the overlap-add method because it requires fewer DFTs, namely 5 DF'Ts for the FDAF configuration using the overlapsave method and 7 DFTs for the overlap-add configuration. At first sight this is a very strange result because the overlapsave and overlap-add methods have equal complexity for fixed coefficient filters.

In this paper we will introduce a new FDAF configuration using the overlap-add method which has the same complexity and convergence behavior as the overlap-save configuration given in [1]. After this introducing section we review in section 2 the overlap-add method for fixed filter coefficients, while furthermore a possible implementation is given for time varying filter configurations. In section 3 we give the overlap-add implementation of an FDAF with 7 DFTs, as proposed in [1]. In section 4 we treat the overlap-add method in further detail. Using these results we introduce a new overlap-add FDAF. In the last section conclusions are given.

\section{Overlap-add method}

As we mentioned before two important operations in AF configurations are convolution and correlation. Because of their similarity we will only deal with the convolution operation using the overlap-add method in this section. First we will shortly review this method for fixed filter coefficients. 

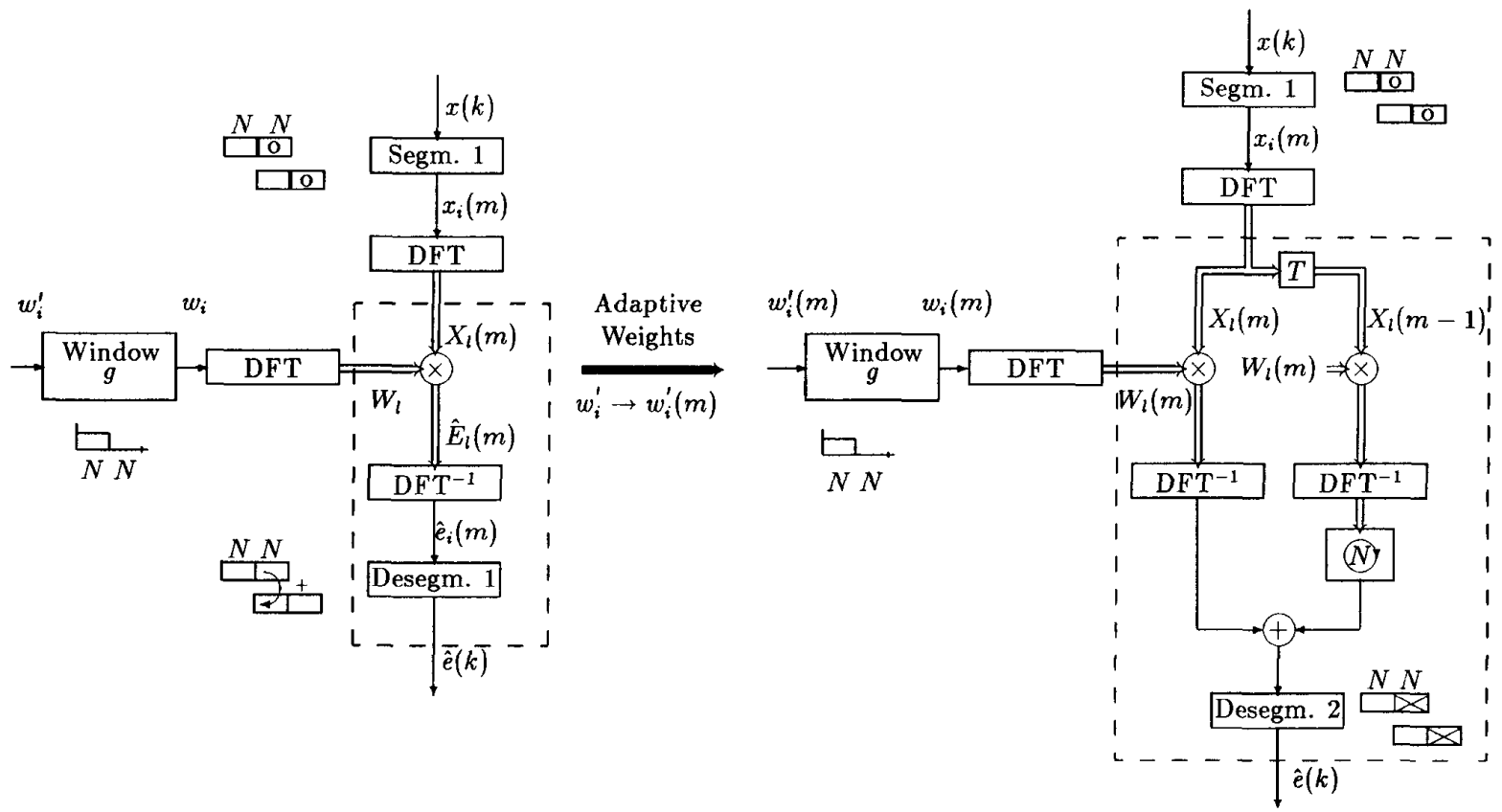

Fig. 2 a Overlap-add method performed in frequency domain.

$\mathrm{b}$ Implementation of Fig. $2 a$ in adaptive filter configurations.

After that we will give the overlap-add implementation of a convolution for time varying filter configurations, which is used in [1] for the FDAF. Fig.2a depicts the overlap-add method for fixed filter coefficients, which is performed in frequency domain using Discrete Fourier Transforms (DFTs). In all figures signal paths with double lines refer to paths in frequency domain while single lines refer to time domain signal paths. Furthermore we choose for simplicity the overlap length of $L$ samples and the number $L^{\prime}$ of augmented zero samples equal to the length $N$ of the impulse response $w_{i}^{\prime}$. In general we can choose $L$ and $L^{\prime}$ more freely. The overlapadd method to calculate the linear convolution between the (infinitely long) input signal $x(k)$ and the (finite) impulse response $w_{i}^{\prime}$ is as follows (see Fig. 2a):

- Augment the impulse reponse $w_{i}^{\prime}$ with $N$ zeros (window $g$ ) and transform this to frequency domain in $W_{l}(m)$ with $l=0,1, \cdots, 2 N-1$.

After that for every iteration $m(=0,1,2, \cdots)$ :

- Select $N$ new samples from the input signal $x(k)$, and augment these by $N$ zeros (segm1), to generate a block

$$
x_{i}(m)= \begin{cases}x(m N+i) & i=0,1, \cdots, N-1 \\ 0 & i=N, \cdots, 2 N-1 .\end{cases}
$$

- Transform this block to frequency domain which results in the frequency bins $X_{l}(m)$ with $l=0,1, \cdots, 2 N-1$.
- The convolution is performed in frequency domain by multiplying $X_{l}(m)$ by $W_{l}$ for $l=0, \cdots, 2 N-1$.

- This result is transformed back to time domain by the inverse $\mathrm{DFT}\left(\mathrm{DFT}^{-1}\right)$ which results in $\hat{e}_{i}(m)$ with $i=0,1, \cdots, 2 N-1$.

- By performing a desegmentation (desegm1) the output signal $\hat{e}(k)$, with $k=m N+i$, is calculated as:

$\hat{e}(m N+i)=\hat{e}_{i}(m)+\hat{e}_{i+N}(m-1)$ for $i=0, \cdots, N-1$.

In $\mathrm{AF}$ configurations the adaptive weights are updated every iteration which implies that they are time variant. In those situations the addition of $\hat{e}_{i}(m)$ and $\hat{e}_{i+N}(m-1)$, as given in equation (2), is not allowed. A possible implementation of the overlap-add method in AF configurations is given in Fig. $2 \mathrm{~b}$. We note here that the box ' $T$ ' in this figure implies a parallel delay over one iteration of $2 N$ frequency bins. The multiplication in frequency domain of $W_{l}(m)$ has to be carried out separately with $X_{l}(m)$ and with the previous value $X_{l}(m-1)$. The result of $W_{l}(m) \cdot X_{l}(m-1)$ in time domain is circularily shifted over $N$ samples to the left and is added to the result of $W_{l}(m) \cdot X_{l}(m)$ in time domain which gives a block that contains $N$ linear convolution samples at the first $N$ places. The desegmentation box (desegm. 2) gives the output signal $\hat{e}(k)$ by taking the first $N$ samples of the block. 


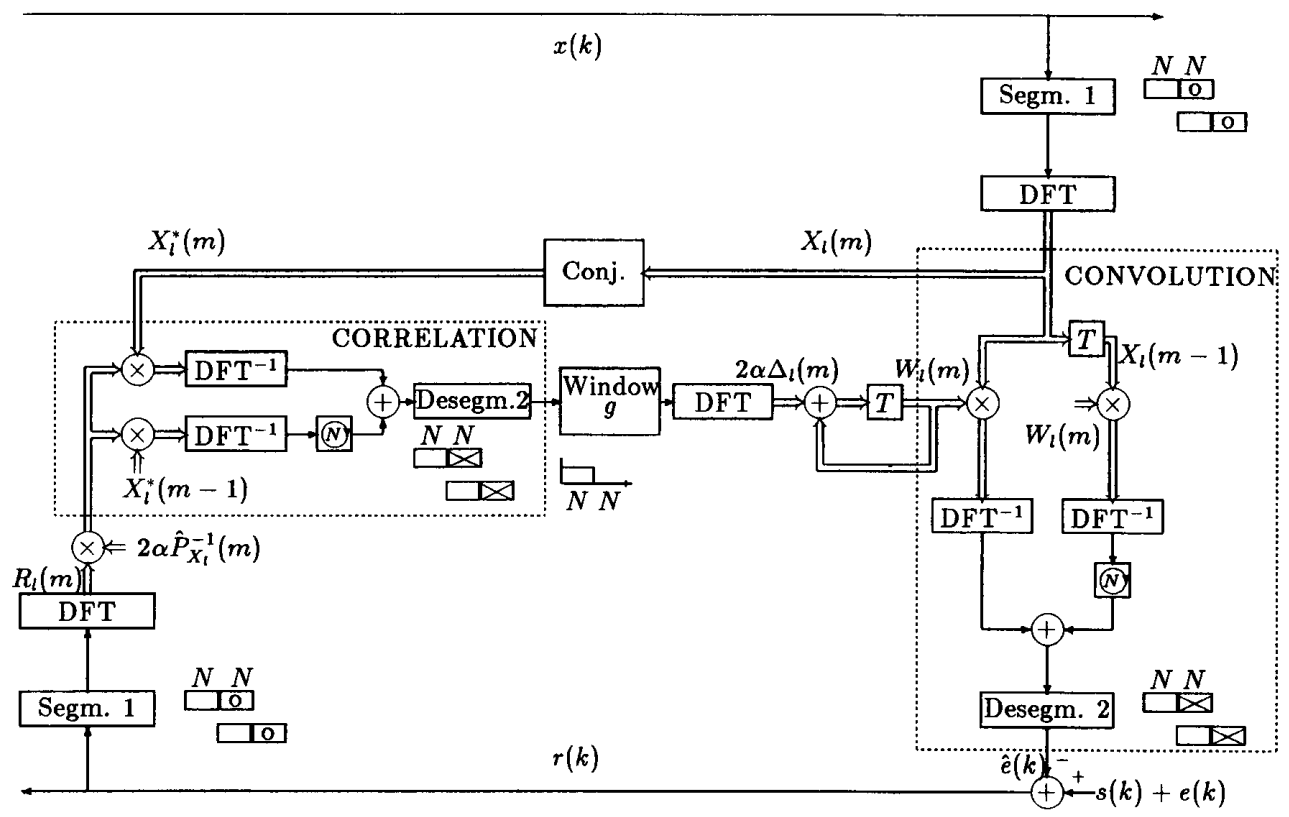

Fig. 3 Overlap-add implementation of an FDAF with 7 DFT's.

\section{Overlap-add FDAF (7 DFTs)}

Using the results of section 2 and refering to [1] (fig.4, page 1080), we give in Fig. 3 an overlap-add implementation of an FDAF. The convolution is performed in the dashed box 'CONVOLUTION'. Furthermore, as mentioned before, the correlation of an 'infinite' signal with a 'finite' sequence can be calculated in a similar way as the convolution. For this we need the complexe conjugate $X_{i}^{*}(m)$ of the input signal while the 'finite' sequence is equal to $N$ samples of $r(k)$. The correlation is performed in the dashed box 'CORRE$L A T I O N$ '. The window $g$ is used to take the first $N$ samples and augment these with $N$ zeros. It is obvious that the operations of the desegmentation (desegm2) and the window $g$ both can be combined in window $g$. Furthermore we see from Fig. 3 that this FDAF configuration contains 7 DFTs.

By decorrelating the input signal the convergence speed can be accelerated [2]. This can be accomplished by normalizing the input power spectrum. In Fig. 3 this is done by multiplying the frequency bins $R_{l}(m)$ of the residual signal by the inverse of an estimate of the $l^{\text {th }}$ input power spectral bin $\hat{P}_{X_{l}}^{-1}(m)$. Because this power normalization is beyond the scope of this paper we will not go into further detail. The update algorithm, for each frequency bin $l$, is now given by:

$$
W_{l}(m+1)=W_{l}(m)+2 \alpha \Delta_{l}(m)
$$

where $\alpha$ is the adaptation constant and $\Delta_{l}(m)$ is an estimate of the (negative) gradient.

\section{New overlap-add FDAF (5 DFTs)}

To introduce the new structure we will first take a closer look to the overlap-add method separately. We will show here that the structure of Fig. 2b can be realized with less DFTs. This simplified structure is given in Fig. 4.

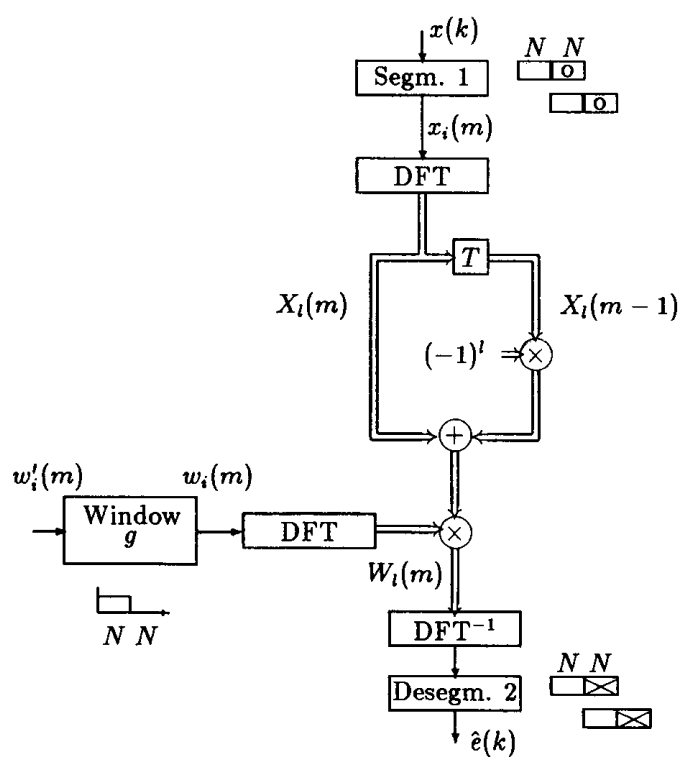

Fig. 4 Overlap-add method of Fig. $2 b$ with circular shift and addition performed in frequency domain. 
First we will use the property that the cicular shift over $N$ samples in time domain (Fig. 2b) is equal to multiplication in frequency domain by $\mathrm{e}^{-\mathrm{j} \theta l N}=\mathrm{e}^{-\mathrm{j} \frac{2 \pi}{2 N} l N}=(-1)^{l}$. Besides that the addition of Fig. $2 \mathrm{~b}$ can be done in frequency domain, which saves one DFT $^{-1}$. Furthermore we can change places of the multiplications and addition. This results in Fig. 4, which is an equivalent of Fig. 2b. We note here that in Fig.4 only one DFT ${ }^{-1}$ is needed, whereas we needed two of them in fig.2b.

Using the above results in the dashed boxes of the FDAF configuration of Fig. 3 leads to the new FDAF configuration given in Fig.5. This new overlap-add implementation contains 5 DFTs whereas the original one (Fig.3) which was derived from $[1]$ contains 7 DFTs.

\section{Conclusions}

In this paper we introduced a new FDAF configuration using the overlap-add method which has 'equivalent' complexity as the FDAF using the overlap-save method ([1], fig.2, $\mathrm{p}$ 1078). Furthermore it can be shown very easily that the convergence properties of both structures are the same.
Another possibility to reduce the number of DFTs of Fig. 3 is to assume that $W_{l}(m) \approx W_{l}(m-1)$, which will be reasonably true for small adaptation constant $\alpha$. Using this assumption it is possible to simplify the structure of Fig. 3 to an 'overlap-add FDAF' with 5 DFTs. However the convergence properties of this scheme will be worse than the FDAF as given in Fig. 5.

As a final comment we mention that, in the FDAF configuration of Fig. 5, we can further reduce the number of DFTs to three by using the 'window techniques' as introduced in [2].

\section{References}

[1] G.A. Clark, S.R. Parker, S.K. Mitra, 'A unified approach to time- and frequency-domain realization of FIR adaptive digital filters', IEEE Trans. on ASSP, vol. ASSP-31, no.5, Oct. 1983, pp 1073-1083 .

[2] P.C.W. Sommen, P.J. van Gerwen, H.J. Kotmans and A.J.E.M. Janssen, 'Convergence Analysis of a Frequency-Domain Adaptive Filter with Exponential Power Averaging and Generalized Window Function', IEEE Trans. on CAS, vol. CAS-34, no.7, july 1987, pp 788-798

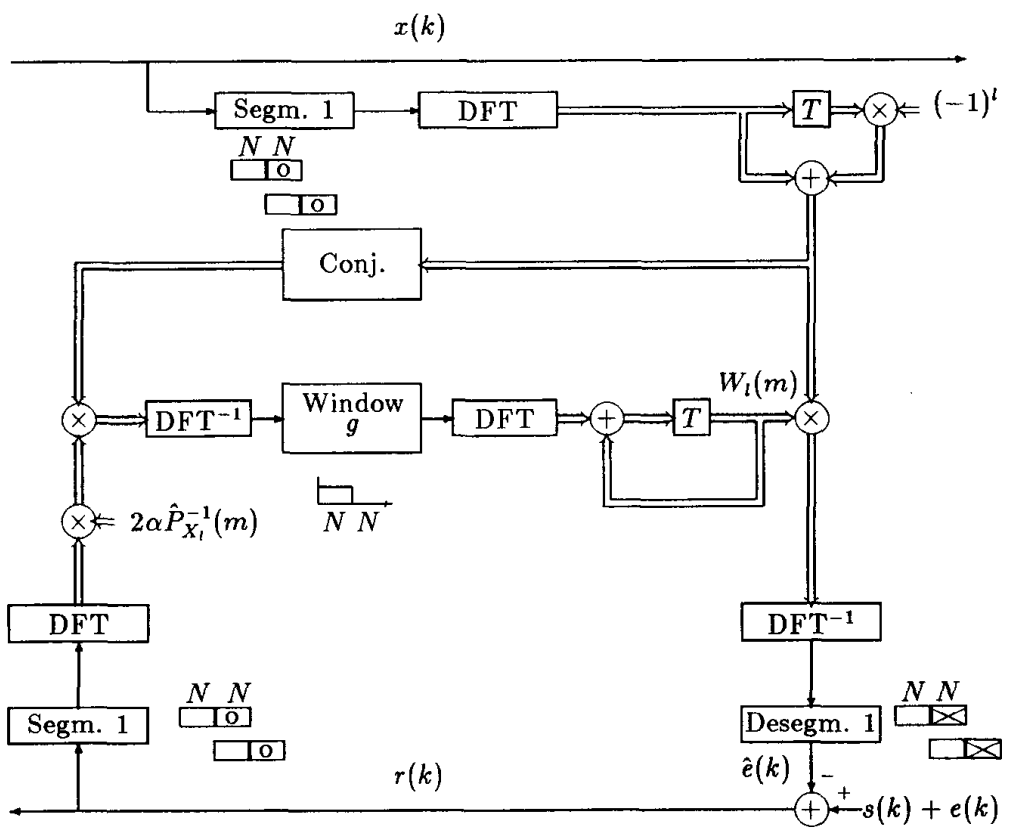

Fig. 5 New overlap-add FDAF with 5 DFTs. 